University of Nebraska - Lincoln

DigitalCommons@University of Nebraska - Lincoln

2-7-2003

\title{
Backscattering of an Intense Laser Beam by an Electron
}

Fei He

University of Michigan, Ann Arbor

Y.Y. Lau

University of Michigan, Ann Arbor

Donald P. Umstadter

University of Nebraska-Lincoln, donald.umstadter@unl.edu

Richard Kowalczyk

University of Michigan, Ann Arbor

Follow this and additional works at: https://digitalcommons.unl.edu/physicsumstadter

Part of the Physics Commons

He, Fei; Lau, Y.Y.; Umstadter, Donald P.; and Kowalczyk, Richard, "Backscattering of an Intense Laser Beam by an Electron" (2003). Donald Umstadter Publications. 19.

https://digitalcommons.unl.edu/physicsumstadter/19

This Article is brought to you for free and open access by the Research Papers in Physics and Astronomy at DigitalCommons@University of Nebraska - Lincoln. It has been accepted for inclusion in Donald Umstadter Publications by an authorized administrator of DigitalCommons@University of Nebraska - Lincoln. 


\title{
Backscattering of an Intense Laser Beam by an Electron
}

\author{
Fei He, Y. Y. Lau, Donald P. Umstadter, and Richard Kowalczyk \\ Department of Nuclear Engineering and Radiological Sciences, University of Michigan, Ann Arbor, Michigan 48109-2104
}

(Received 17 June 2002; published 4 February 2003)

\begin{abstract}
We present a novel, simple asymptotic expansion for the spectrum of radiation that is backscattered from a laser by a counterpropagating (or copropagating) electron. The solutions are presented in such a way that they explicitly show the relative merit of using an intense laser and of an energetic electron beam in $\mathrm{x}$-ray production in the single particle regime. Simple scaling laws are given.
\end{abstract}

DOI: 10.1103/PhysRevLett.90.055002

PACS numbers: 41.60.- $\mathrm{m}, 42.55 . \mathrm{Vc}$

Recent advances in tabletop, ultrahigh intensity lasers have led to significant renewed interest in the classic problem of Thomson and Compton scattering. An important current application of these scattering processes is the generation of ultrashort-pulse-duration $\mathrm{x}$ rays. For example, electrons with only $100 \mathrm{MeV}$ energy can upshift a $1 \mathrm{eV}$ photon to an energy of $50 \mathrm{keV}$, which is of interest not only as a coherent probe with atomic-scale spatial resolution, but also as a medical diagnostic. Several proposals have been made to build a "gamma-gamma" collider for high-energy physics experiments, in which $200-\mathrm{GeV}$ gamma rays are generated by Compton scattering of $1-\mathrm{eV}$ photons from $250-\mathrm{GeV}$ energy electron beams. Colliding such energetic photons to create particles through the inverse process has advantages over direct particle collisions because of reduced beamstrahlung and disruption. The results of numerous experiments [1-6], theories [7-20], and reviews [21,22] related to these topics have been published.

In this paper, we revisit the classical theory for the backscattered radiation from a laser by a counterpropagating electron. While analytic and numeric solutions to this problem have been obtained many times in the past, the novel aspects of the present work include a sharp delineation of the role of the intense laser and of the electron's kinetic energy in the production of high harmonics. For example, we find that the relative shape of the backscatter spectrum depends only on the laser amplitude but is independent of the electron energy, that a high power laser does not necessarily produce high power radiation or overwhelmingly favorable frequency upshift, and that very significant frequency up-shift is achieved most effectively by the use of an energetic electron beam. These findings are consistent with some conventional notions, while contradicting others. They are based on the closed form solutions of the backscattered spectrum that we obtained for arbitrary laser intensity and arbitrary electron energy (including zero), together with the simple asymptotic expressions in the various regimes. Thus, we provide a unifying theme for this scattering problem from a low to an ultrahigh power laser, and from a low to an ultrahigh energy electron, both jointly and separately.

055002-1 0031-9007/03/90(5)/055002(4)\$20.00
Consider a linearly polarized electromagnetic wave whose electric and magnetic fields are given by, respectively, $\boldsymbol{E}=\boldsymbol{x} E_{0} \cos \left(\omega_{0} t-k_{0} z\right)$ and $\boldsymbol{B}=\boldsymbol{y} E_{0} \cos \left(\omega_{0} t-\right.$ $k_{0} z$ ) in Gaussian units, where $\omega_{0}$ is the laser frequency, $k_{0}=\omega_{0} / c$, and $\boldsymbol{x}, \boldsymbol{y}$ are the unit vectors. Unless otherwise stated, we shall normalize time by $1 / \omega_{0}$, distance by $1 / k_{0}$, and velocity by $c$. In these units, the electric field strength is measured by the dimensionless parameter, $a=e E_{0} / m \omega_{0} c$. All calculations are done in the lab frame. Radiation damping is ignored.

The relativistic Lorentz force law, $d(\gamma \boldsymbol{\beta}) / d t=a(\boldsymbol{x}+$ $\boldsymbol{\beta} \times \boldsymbol{y}) \cos (t-z)$, subject to the general initial conditions at time $t=0$, i.e., $x=0, y=0, z=z_{\text {in }}, \beta_{x}=\beta_{x 0}, \beta_{y}=$ $\beta_{y 0}$, and $\beta_{z}=\beta_{z 0}$, may be solved parametrically in terms of $\theta=t-z$, the phase of the wave $[8-12,17,20]$. Here $\boldsymbol{\beta}=\left(\beta_{x}, \beta_{y}, \beta_{z}\right)$ is the electron velocity (in units of $c), \gamma=\left(1-|\boldsymbol{\beta}|^{2}\right)^{-1 / 2}$, and $\boldsymbol{\beta}_{0}=\left(\beta_{x 0}, \beta_{y 0}, \beta_{z 0}\right)$ is the unperturbed velocity of the electron $(a=0$ limit $)$. In the lab frame, the electron motion is periodic with period $T$, and the electron's net displacement over this period is $\boldsymbol{r}_{0}$. Both $T$ and $\boldsymbol{r}_{0}$ depend on the initial phase, $\theta_{\text {in }}=-z_{\text {in }}[9,20]$.

The energy radiated by the electron in the direction of the unit vector $\boldsymbol{n}$, per unit solid angle $\Omega$ in the far field, per unit frequency $\omega$, is given by (in dimensional form [23])

$$
\begin{gathered}
\frac{d^{2} I}{d \Omega d \omega}=\frac{e^{2} \omega^{2}}{4 \pi^{2} c}|\mathbf{n} \times \mathbf{F}(\omega)|^{2}, \\
\mathbf{F}(\omega)=\int_{-\infty}^{\infty} d t \boldsymbol{\beta}(t) e^{i \omega[t-\mathbf{n} \cdot \mathbf{r}(t) / c]} \\
=\sum_{m=-\infty}^{\infty} \mathbf{F}_{m} \delta\left(\omega-m \omega_{1}\right),
\end{gathered}
$$

where $\delta$ is the Dirac delta function. In Eq. (2), the radiation spectrum is at integer harmonics of the frequency $\omega_{1}$, which is related to the laser frequency $\omega_{0}$ in terms of the dimensionless quantities by $\omega_{1} / \omega_{0}=$ $2 \pi /\left(T-\mathbf{n} \cdot \mathbf{r}_{\mathbf{0}}\right)[10,17,20]$, and $\mathbf{F}_{m}$ is the dimensionless vector, 


$$
\begin{aligned}
\mathbf{F}_{m} & =\frac{\omega_{1}}{\omega_{0}} \int_{\theta_{\mathrm{in}}}^{\theta_{\mathrm{in}}+2 \pi} d \theta \frac{\boldsymbol{\beta}}{1-\beta_{z}} e^{i m 2 \pi h(\theta),} \\
h(\theta) & =\frac{t(\theta)-\mathbf{n} \cdot \mathbf{r}(\theta)}{T-\mathbf{n} \cdot \mathbf{r}_{0}} .
\end{aligned}
$$

Both $\omega_{1}$ and $\mathbf{F}_{m}$ depend on $\omega_{0}, a, \theta_{\text {in }}, \boldsymbol{\beta}_{0}$, and $\mathbf{n}$.

We henceforth focus on the special case of backscattered radiation $(\boldsymbol{n}=-\boldsymbol{z})$ by an electron that has the following initial conditions: $\theta_{\text {in }}=0, \beta_{x 0}=0, \beta_{y 0}=0$, and $\beta_{z 0}$ may be negative (electron counterpropagates against laser), zero (electron is initially at rest), or positive (electron copropagates with laser). In this case, one finds

$$
\frac{\omega_{1}}{\omega_{0}}=\left(\frac{2}{2+a^{2}}\right)\left(\frac{1-\beta_{z 0}}{1+\beta_{z 0}}\right)=\left(\frac{2}{2+a^{2}}\right) \gamma_{0}^{2}\left(1-\beta_{z 0}\right)^{2},
$$

where $\gamma_{0}=\left(1-\beta_{z 0}^{2}\right)^{-1 / 2}$. The power, $p_{m}$ (in erg/s), radiated at the harmonic frequency $\omega=m \omega_{1}$ per unit solid angle in the $\boldsymbol{n}=-\boldsymbol{z}$ direction is given by [cf. Eq. (1)]

$$
p_{m}=\frac{e^{2} m^{2} \omega_{1}^{2}}{4 \pi^{2} c}\left|\mathbf{n} \times \mathbf{F}_{m}\right|^{2}=\frac{A}{\gamma_{0}^{2}\left(1-\beta_{z 0}\right)^{2}}\left(\frac{\omega_{1}}{\omega_{0}}\right)^{4} s_{m}
$$

where $A=e^{2} \omega_{0}^{2} / 4 \pi^{2} c=0.69[\lambda /(1 \mu \mathrm{m})]^{-2} \mathrm{erg} / \mathrm{s}$, and $\lambda$ is the laser wavelength. In Eq. (5), we find that $s_{m}=0$ for $m=0, \pm 2, \pm 4, \ldots$ [Figs. 1(a) and 1(b)]. For $m=$ $\pm 1, \pm 3, \pm 5, \ldots$, one finds from Eqs. (3) and (5) the closed form solution,

$$
\begin{gathered}
s_{m}=(a \pi)^{2} m^{2}\left[J_{(m-1) / 2}(m \kappa)-J_{(m+1) / 2}(m \kappa)\right]^{2} \\
\kappa=\frac{a^{2}}{2\left(a^{2}+2\right)},
\end{gathered}
$$

where $J_{n}(x)$ is the Bessel function of the first kind of order $\nu$. The factor $s_{m}$ appears in Esarey et al. [11]. It also appears in the quantity " $[J J]$ " or " $F_{m}(K)$ " in the FEL/ synchrotron light literature if one replaces $a$ by the undulator/wiggler parameter $K$ [24-27]. The normalized distribution of $s_{m}$ is shown in Figs. 1(a)-1(d) for increasing values of $a$. The maximum values of $s_{m}$, occurring at $m=M$ with a value $s_{M}$, are shown in Fig. 2. It is easy to show that $s_{m}=s_{-m}$ for all odd integers $m$.

The electron's unperturbed velocity enters $p_{m}$ in Eq. (5) only through the factor $\left(\omega_{1} / \omega_{0}\right)^{4} /\left[\gamma_{0}\left(1-\beta_{z 0}\right)\right]^{2}$. The dimensionless quantities $s_{m}$ and $\kappa$ depend only on the laser intensity, $a$. In other words, the relative shape of the backscatter spectrum by a single electron depends only on the laser intensity and is independent of the electron's unperturbed velocity, as shown in Figs. 1 and 2. Thus, the exact solutions (5) and (6) provide an unambiguous separation of the roles of the two drives, the laser and the electron's unperturbed velocity, in producing backscatter radiation.

For $a \leq 0.3$, the $m= \pm 1$ components contain essentially all backscattered radiation [Fig. 1(a)], and Eqs. (6)
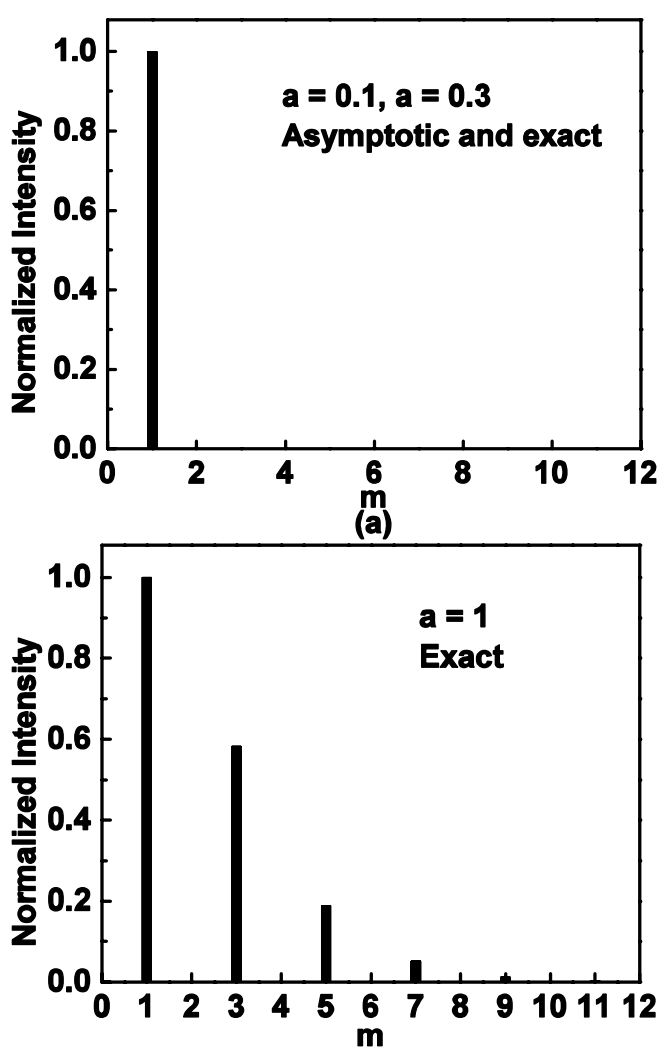

(b)

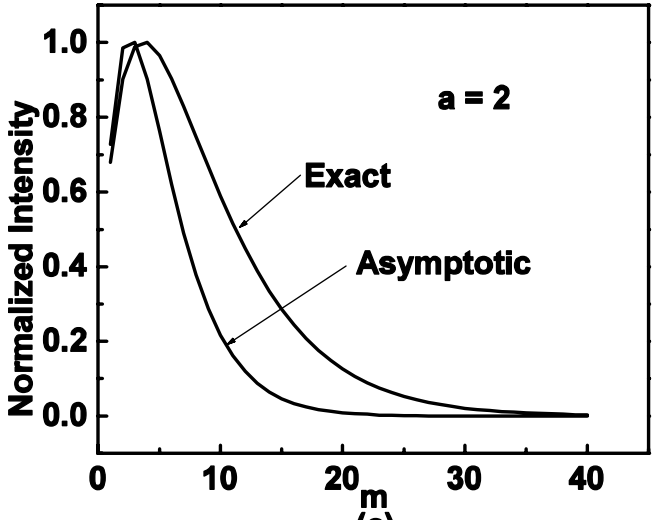

(c)

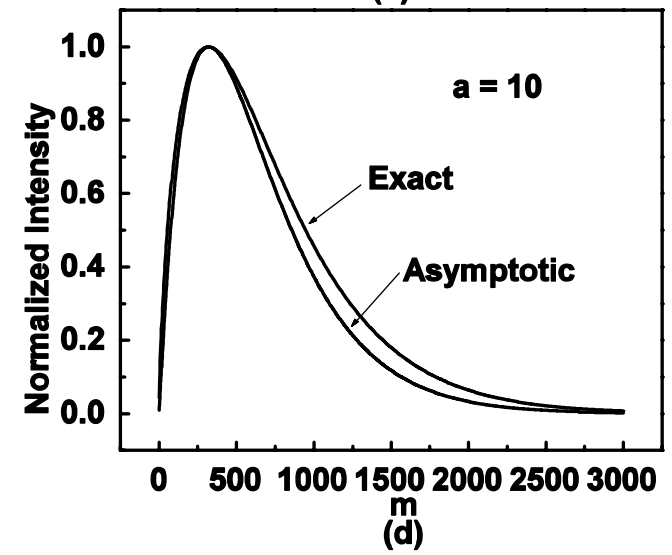

FIG. 1. Normalized spectral distribution of $s_{m}$, at frequency $\omega=m \omega_{1}$. Here $s_{m}$ is normalized with respect to the maximum value $s_{M}$, occurring at $m=M$. 

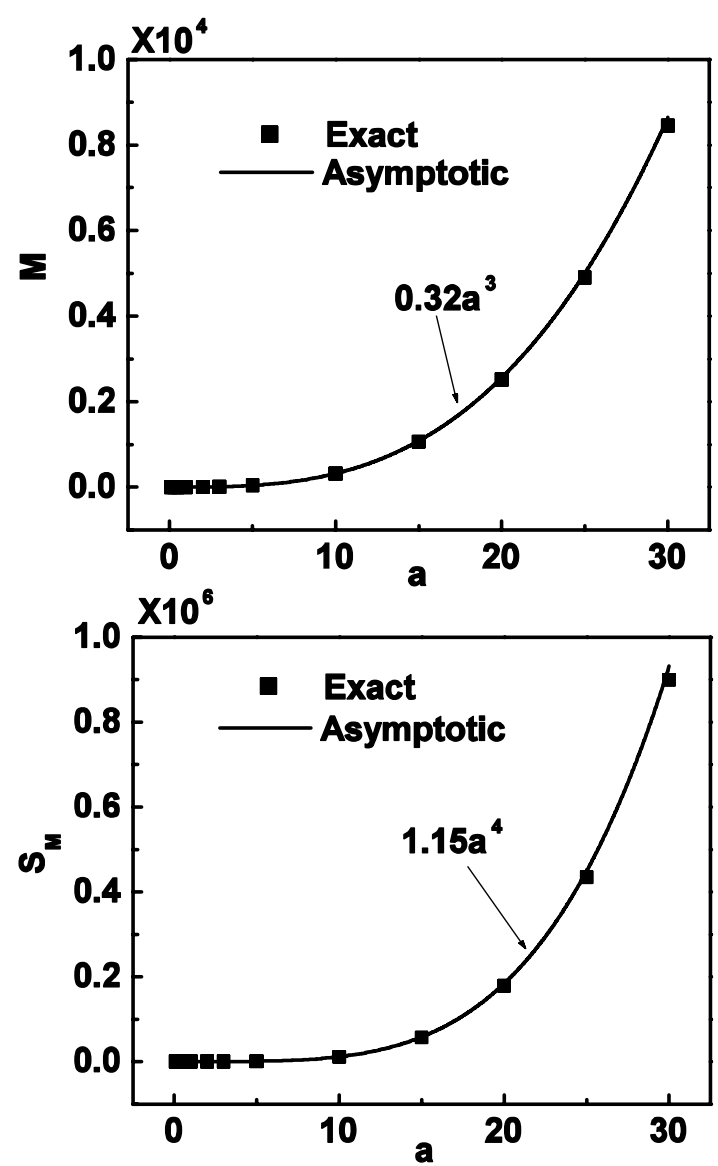

FIG. 2. Numerical values of $M$ (top) and $s_{M}$ (bottom) at various values of $a$.

and (7) give $s_{1} \cong a^{2} \pi^{2}$ for $a^{2} \ll 1$. For $a=1, s_{m}$ is appreciable only for the first few odd numbers of $m$ [Fig. 1(b)]. For $a \geq 2, s_{m}$ spreads out to larger values of $m$ [Figs. 1(c) and 1(d)]. A saddle point calculation gives, for $a^{2} \gg 1$,

$$
\begin{gathered}
s_{m} \approx s_{M}(|m| / M) e^{-(|m|-M) / M}, \\
M \approx 0.32 a^{3}, \quad s_{M} \approx 1.15 a^{4} .
\end{gathered}
$$

The asymptotic formula (8) is shown in Figs. 1(c) and 1(d) for comparison. The asymptotic formula (9) is shown in Fig. 2. Once more, the frequency component $\omega=M \omega_{1}$ contains the highest backscatter power.

In terms of the laser frequency, the frequency component $\omega=N \omega_{0}$ would contain the highest backscattered power, where $N=M \omega_{1} / \omega_{0}$. Upon using Eq. (4), we obtain

$$
\begin{array}{ll}
N=\left(\frac{2}{2+a^{2}}\right) \gamma_{0}^{2}\left(1-\beta_{z 0}\right)^{2}, & a \leq 1, \\
N \approx 0.64 a \gamma_{0}^{2}\left(1-\beta_{z 0}\right)^{2}, & a \geq 3 .
\end{array}
$$

Equation (10) clearly shows the merit of using an energetic electron beam, over that of using an intense laser, in the generation of high frequency backscattered radiation.
It also shows the familiar Doppler up-shift proportional to $\gamma_{0}^{2}$ as $\beta_{z 0}$ approaches -1 . The harmonic number of the laser frequency, $N$, at which maximum backscattered power occurs is shown in Fig. 3 (top). Note that $N$ is linearly proportional to $a$, instead of $a^{3}$, for large $a[9,20]$.

The total backscattered power, $P_{T}$ (in ergs/s), per unit solid angle in the $\boldsymbol{n}=-\boldsymbol{z}$ direction is then given by $P_{T}=$ $\Sigma p_{m}$ where the sum is taken over all odd values of $m$ [Fig. 1(b)]. The following approximate values of $P_{T}$ are obtained for small and large values of $a$,

$$
\begin{aligned}
P_{T} & \approx p_{-1}+p_{1}=2 p_{1} \approx 13.7 a^{2} \frac{\gamma_{0}^{6}\left(1-\beta_{z 0}\right)^{6}}{(\lambda / 1 \mu \mathrm{m})^{2}} \mathrm{erg} / \mathrm{s}, \\
a & \leq 0.3 \\
P_{T} & \approx \frac{1}{2} \int_{-\infty}^{\infty} d m p_{m} \approx \frac{11.1}{a} \frac{\gamma_{0}^{6}\left(1-\beta_{z 0}\right)^{6}}{(\lambda / 1 \mu \mathrm{m})^{2}} \mathrm{erg} / \mathrm{s}, \\
a & \geq 3 .
\end{aligned}
$$
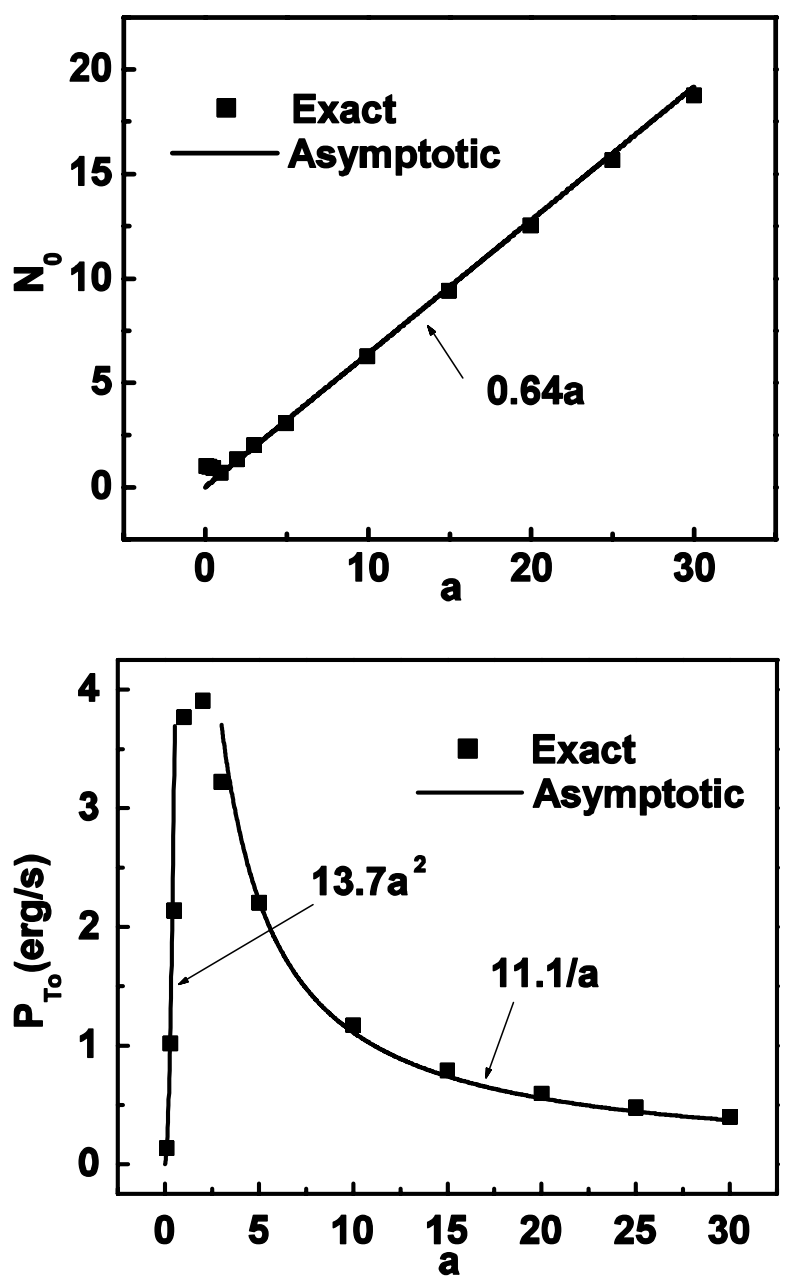

FIG. 3. (Top) The harmonic number of the laser frequency, $N_{0}$, at which maximum backscatter occurs for the special case $\beta_{z 0}=0$. For nonzero $\beta_{z 0}, N=\gamma_{0}^{2}\left(1-\beta_{z 0}\right)^{2} N_{0}$. (Bottom) The total backscattered power, $P_{T 0}$, per unit solid angle for the special case $\beta_{z 0}=0$, and laser wavelength $\lambda=1 \mu \mathrm{m}$. For other values of $\beta_{z 0}$ and $\lambda, P_{T}=P_{T 0} \gamma_{0}^{6}\left(1-\beta_{z 0}\right)^{6} /(\lambda / 1 \mu \mathrm{m})^{2}$. 
In writing Eq. (11a), we use the fact that only the $m=+1$ and -1 components dominate for $a<1$ [Fig. 1(a)]. In writing Eq. (11b), we pretend that the discrete variable $m$ becomes continuous for $a>3$ [Figs. 1(c) and 1(d)], and we use Eq. (8) to approximate $p_{m}$ [cf. Eq. (5)]. The factor of $\frac{1}{2}$ in front of the integral in Eq. (11b) accounts for the fact that $p_{m}=0$ for all even values of $m$ (i.e., only odd values of $m$ contribute to $P_{T}$ ). The approximate solutions (11a) and (11b) are shown in Fig. 3 (bottom) for comparison.

Note the unusual scaling of $P_{T}$, which is proportional to $\gamma_{0}^{6}$ according to Eqs. (11a) and (11b) in the highly relativistic limit $\beta_{z 0} \rightarrow-1$. Such a rapid increase in radiated power with the electron energy $\left(\gamma_{0}\right)$ is hardly surprising in the classical theory of radiation of electron [23]. Note further that Eq. (11b) clearly shows that using an ultra-intense laser (large $a$ ) does not necessarily yield the brightest backscatter radiation, as it gives the total backscatter power per unit solid angle.

Our highly idealized model of laser backscatter by a single electron ignores important effects such as electron beam emittance, finite beam and laser pulselengths, spectral linewidth and angular distributions, all of which have been studied extensively for synchrotron light/free electron laser [24-29]. A great number of these effects can be directly translated in a generalization of the present study, as can the collective effects [30]. Likewise, the asymptotic solutions given in this paper may also be immediately applied to free electron lasers because $s_{m}$ and $[J J]$ or $F_{m}(K)$ are basically the same function, as indicated above.

In conclusion, we have constructed simple scaling laws for the spectrum of the backscattered radiation of an electron by an intense laser, according to the classical theory. These solutions suggest that an intense laser with $a=O(1)$, together with a most energetic counterpropagating electron beam, would produce the combined largest frequency up-shift and brightest backscatter power.

This work is supported by ONR, DoE (Chemical Sciences, Geosciences and Biosciences Division of the Office of Basic Energy Sciences), and NSF.

[1] B. G. Englert et al., Phys. Rev. A 28, 1539 (1983).

[2] R.W. Schoenlein et al., Science 274, 236 (1996).

[3] D. D. Meyerhofer et al., J. Opt. Soc. Am. B 13, 113 (1996).

[4] S.-Y. Chen, A. Maksimchuk, and D. Umstadter, Nature (London) 396, 653-655 (1998).

[5] C. Bamber et al., Phys. Rev. D 60, 092004 (1999).
[6] S. Y. Chen, A. Maksimchuk, E. Esarey, and D. Umstadter, Phys. Rev. Lett. 84, 5528-5531 (2000).

[7] Vachaspati, Phys. Rev. 128, 664 (1962).

[8] E. S. Sarachik and G. T. Schappert, Phys. Rev. D 1, 2738 (1970).

[9] J.E. Gunn and J.P. Ostriker, Astrophys. J. 165, 523 (1971).

[10] A. K. Puntajer and C. Leubner, Phys. Rev. A 40, 279 (1989).

[11] E. Esarey, S. K. Ride, and P. Sprangle, Phys. Rev. E 48, 3003 (1993).

[12] P. Sprangle, A. Ting, E. Esarey, and A. Fisher, J. Appl. Phys. 72, 5032 (1992); A. Ting et al., ibid. 78, 575 (1995).

[13] I. Christov et al., Opt. Commun. 148, 75 (1998).

[14] I. Yu. Kostyukov, Phys. Rev. E 59, 1122 (1999).

[15] A. E. Kaplan and P. L. Shkolnikov, Phys. Rev. Lett. 88, 074801 (2002); 89, 199502 (2002); G. Stupakov and M. Zolotorev, ibid. 89, 199501 (2002).

[16] F.V. Hartemann, Phys. Plasmas 5, 2037 (1998); Phys. Rev. E 64, 016501 (2001).

[17] Y. I. Saliman and F. H. M. Faisal, Phys. Rev. A 54, 4383 (1996); 55, 3964 (1997); J. Phys. A 31, 1319 (1998).

[18] P. Panek, J. Z. Kaminski, and F. Ehlozky, Phys. Rev. A 65, 022712 (2002).

[19] U. Mohideen et al., J. Opt. Soc. Am. B 9, 2190 (1992).

[20] F. He, Y. Y. Lau, D. Umstadter, and T. Strickler, Phys. Plasmas 9, 4325 (2002); 10, 327(E) (2003).

[21] Proceedings of Workshop on Compton Scattering, edited by I.V. Pogorelsky [Nucl. Instrum. Methods Phys. Res., Sect. A 455, No. 1 (2000), and references therein].

[22] F.V. Hartemann, High-Field Electrodynamics (CRC Press, Boca Raton, 2001), and references therein.

[23] J. D. Jackson, Classical Electrodynamics (Wiley, New York, 1962), p. 480.

[24] K. J. Kim, in Physics of Particle Accelerators, edited by M. Month and M. Dienes, AIP Conf. Proc. No. 184 (AIP, New York, 1989), p. 565; Nucl. Instrum. Methods Phys. Res., Sect. A 250, 396 (1986); Opt. Eng. (Bellingham, Wash.) 34, 342 (1995).

[25] S. Krinsky et al., in Handbook on Synchrotron Radiation, edited by E. E. Koch (North-Holland, Amsterdam, 1983).

[26] C. A. Brau, Free-Electron Lasers (Academic, San Diego, 1990).

[27] P. G. O'Shea and J. B. Murphy (to be published).

[28] K. J. Kim, Nucl. Instrum. Methods Phys. Res., Sect. A 246, 67 (1986); 246, 71 (1986).

[29] D. Attwood, Soft X-Rays and Extreme Ultraviolet Radiation: Principles and Applications (Cambridge University Press, Cambridge, U.K., 1999).

[30] See, e.g., A. Gover and P. Sprangle, IEEE J. Quantum Electron. QE17, 1196 (1981); L. Schacter, Beam-Wave Interaction in Periodic and Quasi-Periodic Structures (Springer-Verlag, Berlin, 1996). 